Rev Inv Vet Perú 2017; 28(3): 737-742

http://dx.doi.org/10.15381/rivep.v28i3.13365

\title{
Comunicación
}

\section{Características Fisicoquímicas de la Laguna Chauya (Mayo 2015-Abril 2016)}

\author{
Physicochemical Characteristics of the Chauya Lagoon (May 2015-April 2016) \\ Guadalupe Contreras S. ${ }^{1}$, José C. Riofrio-Quijandría ${ }^{2}$
}

\section{Resumen}

La laguna Chauya es un cuerpo de agua ubicado en el distrito de Masisea (Ucayali, Perú), con un área de $34.77 \mathrm{~km}^{2}$. Es un lugar de pesca importante para la flota comercial de Pucallpa y está considerada dentro del área de Conservación Regional de Imiria. El Plan de Manejo Pesquero fue desarrollado en 2008, pero no ha sido objeto de seguimiento. El propósito del presente estudio fue realizar una evaluación de las características fisicoquímicas de la laguna y compararlas con los resultados del estudio inicial. Los resultados revelaron que las características fisicoquímicas del ambiente y del agua se hallaron dentro de lo manifestado por el Plan de Manejo Pesquero, no hallándose diferencias importantes en términos de temperatura ambiente y subsuperficial del agua, $\mathrm{pH}$, amonio, $\mathrm{CO}_{2}$, cloruros, dureza y oxígeno disuelto en agua.

Palabras clave: Chauya; Imiria; plan de manejo pesquero; fisicoquímica; Amazonia peruana

\section{Abstract}

The Chauya is a waterbody located in the district of Masisea (Ucayali, Peru) in an area of $34.77 \mathrm{~km}^{2}$. The lagoon is important for the commercial fishing fleet of Pucallpa and is part of the Regional Conservation of Imiria. The Fisheries Management Plan was developed in 2008, but it has not followed up. The aim of this study was to assess the physicochemical characteristics of the lagoon and its comparison with the initial study. The results showed that the physicochemical characteristics of the environment and

\footnotetext{
${ }^{1}$ Facultad de Medicina Veterinaria, ${ }^{2}$ Facultad de Ciencias Biológicas, Universidad Nacional Mayor de San Marcos, Lima, Perú

${ }^{3}$ E-mail: lupecontreras@hotmail.com
}

Recibido: 23 de noviembre de 2016

Aceptado para publicación: 25 de mayo de 2017 
water were like in the original Fisheries Management Plan, and without significant differences in terms of ambient and water subsurface temperature, $\mathrm{pH}$, ammonia, $\mathrm{CO}_{2}$, chlorides, water hardness, and dissolved oxygen in water.

Key words: Chauya; Imiria; Fisheries Management Plan; physicochemical; Peruvian Amazonia

\section{INTRODUCCIÓN}

El complejo lagunar Chauya (Riofrio et al., 2008) se encuentra dentro del Área de Conservación Regional del Imiria, región Ucayali, Perú (899900-9015000 S y $578600-$ $9009000 \mathrm{~W})$, establecido mediante DS 0062010-MINAM. Riofrio et al. (2008) lo caracterizaron como un cuerpo de agua multitrófico, por presentar áreas en oligotrofia y en eutrofia. Por otro lado, considerando la composición del plancton, Samanez (2008) señala que el cuerpo de agua tiene características de mesotrófico a eutrófico. El complejo tiene un área de $34.77 \mathrm{~km}^{2}$ y de acuerdo a los estudios batimétricos realizados por Ramírez y Velarde (2008), puede ser considerada somera, con una profundidad máxima de $9.7 \mathrm{~m}$.

Con base a los estudios mencionadas y los resultados de evaluación de pesquerías realizados por Bazán y Deza (2008) y Velarde et al. (2008) se llegó a completar el Plan de Manejo Pesquero de la laguna Chauya, aunque su monitoreo no se ha hecho desde su aprobación. En 2015, el Gobierno Regional de Ucayali, a través de su Gerencia de Recursos Naturales, con la finalidad de dar cumplimiento con los monitoreos establecidos, convocó a instituciones que laboran en la región para, mediante trabajos de cooperación, realizar las evaluaciones de la pesca en dicho lugar, así como la toma de información fisicoquímica, a fin de contrastar la información con los resultados de 2007-2008.

El presente trabajo es el resultado de las evaluaciones fisicoquímicas realizadas en 2015-2016.

\section{Materiales y Metodología}

Las salidas de campo se programaron con la finalidad de cubrir el ciclo hidrológico del río Ucayali, en sus cuatro fases (Riofrio, 2002): creciente (enero-marzo), transición a vaciante (abril-junio), vaciante (julio-setiembre) y transición a creciente (octubre-diciembre). Se establecieron tres estaciones fijas de muestreo: Huamuya (592075 S / 9012920 W), Agua Blanca (587797 S / 9011988 W) y Yarinal (0581209 S / 9009150 W) (Figura 1).

El estudio estuvo limitado por motivos presupuestales, disponibilidad de instrumentos y carencia de los kits de análisis. No obstante, se obtuvo la siguiente información: temperatura ambiente y sub-superficial del agua (termómetro protegido), $\mathrm{pH}$ (pHmetro Hanna, rango 0-14), y mediciones sobre concentración de oxígeno disuelto, alcalinidad, dureza y amonio, empleado kits comerciales (LaMotte, EEUU). Los trabajos se ejecutaron entre las 08:00 y 11:00 h en las tres estaciones de muestreo.

\section{Resultados y Discusión}

Se agrupó la data en dos épocas de contraste: creciente y vaciante, ya que la información obtenida no era consistente para trabajarla en las cuatro épocas planificadas. Los resultados de las mediciones ambientales y fisicoquímicas del agua se presentan en el Cuadro 1. Los valores mínimos de cloruro fueron estimados con los valores detectables, ya que se encontraban por debajo del nivel de detección de los equipos. Los registros de 


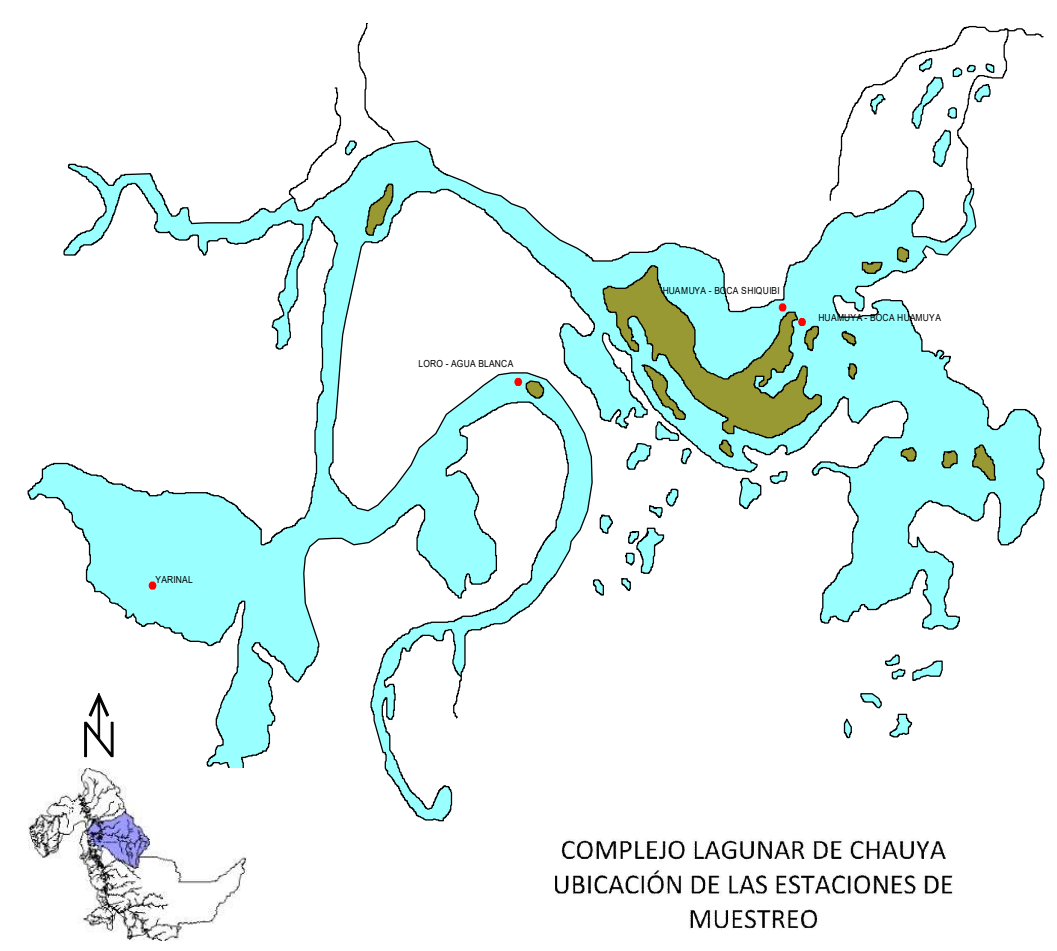

Figura 1. Ubicación de las estaciones fijas de muestreo en la laguna Chauya, región Ucayali, Perú (puntos rojos)

Cuadro 1. Características generales ambientales y fisicoquímicas de la laguna Chauya (20152016)

\begin{tabular}{lccccc}
\hline Parámetro & Promedio & D.E. & Máximo & Mínimo & Rango \\
\hline Temp. ambiental $\left({ }^{\circ} \mathrm{C}\right)$ & 27.7 & 3.1 & 33.5 & 23 & 10.5 \\
$\mathrm{TSS}^{1}$ agua $\left({ }^{\circ} \mathrm{C}\right)$ & 30.1 & 1.2 & 32 & 28.5 & 3.5 \\
$\mathrm{pH}$ & 6.4 & 0.4 & 7.5 & 6 & 1.5 \\
Amonio & 0.6 & 0.2 & 1 & 0.2 & 0.8 \\
Alcalinidad $(\mathrm{mg} \mathrm{CaCO} / \mathrm{l})$ & 46.1 & 21.2 & 120 & 18.5 & 101.5 \\
$\mathrm{CO}_{2}(\mathrm{mg} / \mathrm{l})$ & 11.3 & 4.4 & 25 & 6 & 19 \\
$\mathrm{Cloruro}$ & 30.9 & 11.9 & 56 & 12 & 44 \\
Dureza $(\mathrm{mg} \mathrm{CaCO} / \mathrm{l})$ & 43.8 & 20.6 & 96 & 20 & 76 \\
$\mathrm{O}_{2}(\mathrm{mg} / \mathrm{l})$ & 3.3 & 1.9 & 6.3 & 0.6 & 5.7 \\
\hline
\end{tabular}

${ }^{1}$ Temperatura sub-superficial del agua 
Cuadro 2. Características generales ambientales y fisicoquímicas de la laguna Chauya, según época del año (2015-2016)

\begin{tabular}{lccccccccccc}
\hline & \multicolumn{4}{c}{ Creciente } \\
\cline { 2 - 6 } & Promedio D.E. Máximo Mínimo Rango Promedio D.E. Máximo Mínimo Rango \\
\hline $\begin{array}{l}\text { Temp. ambiental } \\
\left({ }^{\circ} \mathrm{C}\right)\end{array}$ & 27.3 & 2.5 & 32 & 25 & 7 & 28.0 & 3.6 & 33.5 & 23 & 10.5 \\
$\mathrm{TSS}^{1}$ agua $\left({ }^{\circ} \mathrm{C}\right)$ & 30.1 & 1.2 & 32 & 29 & 3 & 30.2 & 1.4 & 32 & 28.5 & 3.5 \\
$\mathrm{pH}$ & 6.4 & 0.5 & 7.5 & 6 & 1.5 & 6.3 & 0.3 & 6.5 & 6 & 0.5 \\
Amonio & 0.6 & 0.2 & 0.8 & 0.2 & 0.6 & 0.7 & 0.3 & 1 & 0.2 & 0.8 \\
Alcalinidad (mg & 50.1 & 28.0 & 120 & 18.5 & 101.5 & 42.2 & 11.9 & 72 & 32 & 40 \\
$\left.\mathrm{CaCO}_{3} / \mathrm{l}\right)$ & 14.0 & 5.3 & 25 & 7 & 18 & 9.3 & 2.1 & 13 & 6 & 7 \\
$\mathrm{CO}_{2}(\mathrm{mg} / \mathrm{l})$ & 28.4 & 15.5 & 56 & 12 & 44 & 33.3 & 6.9 & 38 & 16 & 22 \\
$\mathrm{Cloruro}$ & 46.0 & 8.6 & 56 & 26 & 30 & 42.2 & 26.6 & 96 & 20 & 76 \\
Dureza $\left(\mathrm{mg}_{\mathrm{CaCO} / \mathrm{l})}\right.$ & 3.9 & 2.1 & 6.3 & 0.6 & 5.7 & 2.8 & 1.6 & 6 & 0.6 & 5.4 \\
$\mathrm{O}_{2}(\mathrm{mg} / \mathrm{l})$ & & & & & & & & & & \\
\hline
\end{tabular}

1 Temperatura sub superficial del agua

las características fisicoquímicas se encontraron dentro del rango de valores reportados por Riofrio et al. (2008).

El pH ligeramente ácido y la baja alcalinidad caracterizan un cuerpo de agua negra, además de una baja conductividad y moderada transparencia que no fueron medidas en el estudio (Sioli, 1984). El valor de la dureza inferior a $75 \mathrm{mg} \mathrm{CaCO}_{3} / 1$ la tipifican como aguas blandas (Esteves, 1998) y de acuerdo a la relación entre la alcalinidad y la dureza, se deduce que los bicarbonatos son la principal forma de alcalinidad. La dureza carbonácea, temporal o no permanente, es igual a la dureza total (Romero, 2013). Esto estaría de acuerdo a lo manifestado por Roldán (1992) con respecto al sistema buffer, que indica que el dióxido de carbono libre es importante a un $\mathrm{pH}$ por debajo de 5 , y que el bicarbonato domina en el rango de 7 a 9 y el carbonato en el rango de 10 a 12.

El Cuadro 2 muestra los valores de las mediciones ambientales y fisicoquímicas del agua, según las estaciones de creciente $\mathrm{y}$ vaciante. En general, la alcalinidad, el $\mathrm{CO}_{2}$, la dureza y el $\mathrm{O}_{2}$ presentaron menores valores durante la vaciante, en tanto que los cloruros presentaron valores ligeramente superiores en esta época. Los demás valores fueron similares entre estaciones.

Las características fisicoquímicas y ambientales por estación de muestreo y época del año se presentan en el Cuadro 3.

En la estación de Huamuya, la temperatura ambiental y los cloruros tendieron a subir en la vaciante, en tanto que la alcalinidad, $\mathrm{CO}_{2}$ y dureza del agua fueron mayores durante la creciente. En la estación de Agua Blanca, la temperatura ambiental, los cloruros y dureza del agua tendieron a subir en la vaciante, mientras la alcalinidad, $\mathrm{CO}_{2}$ y oxígeno disuelto en el agua fueron mayores durante la creciente. Por último, en la estación de Yarinal, la alcalinidad fue ligeramente superior durante la vaciante, en total que el $\mathrm{pH}$, $\mathrm{CO}_{2}$, dureza y oxígeno disuelto en el agua más altos en la creciente. 
Cuadro 3. Características ambientales y físicoquímicas en las estaciones de muestreo por época del año (2015-2016)

\begin{tabular}{lcccccccc}
\hline \multirow{2}{*}{ Huamuya } & \multicolumn{4}{c}{ Creciente } & \multicolumn{5}{c}{ Vaciante } \\
\cline { 2 - 9 } & Promedio & D.E. Máximo & Mínimo & Promedio & D.E. Máximo & Mínimo \\
\hline Temp. ambiental $\left({ }^{\circ} \mathrm{C}\right)$ & 26.8 & 1.1 & 27.5 & 26 & 28.5 & 4.3 & 33.5 & 23.5 \\
$\mathrm{TSS}^{1}$ agua $\left({ }^{\circ} \mathrm{C}\right)$ & 29.5 & 0.7 & 30 & 29 & 30.0 & 1.8 & 32 & 28.5 \\
$\mathrm{pH}$ & 6.3 & 0.4 & 6.5 & 6 & 6.3 & 0.3 & 6.5 & 6 \\
Amonio & 0.4 & 0.3 & 0.6 & 0.2 & 0.3 & 0.1 & 0.4 & 0.2 \\
Alcalinidad $\left(\mathrm{mg} \mathrm{CaCO}_{3} / 1\right)$ & 46.0 & 8.5 & 52 & 40 & 34.7 & 2.3 & 36 & 32 \\
$\mathrm{CO}_{2}(\mathrm{mg} / \mathrm{l})$ & 14.0 & 1.4 & 15 & 13 & 9.0 & 2.9 & 13 & 6 \\
$\mathrm{Cloruro}$ & 15.0 & 4.2 & 18 & 12 & 34.0 & 3.5 & 38 & 32 \\
Dureza $(\mathrm{mg} \mathrm{CaCO} / \mathrm{l})$ & 35.0 & 12.7 & 44 & 26 & 23.3 & 5.9 & 32 & 20 \\
$\mathrm{O}_{2}(\mathrm{mg} / \mathrm{l})$ & 3.5 & 0.7 & 4 & 3 & 3.6 & 2.2 & 6 & 1.6 \\
\hline
\end{tabular}

\section{Agua Blanca}

\begin{tabular}{lcccccccc}
\hline Temp. ambiental $\left({ }^{\circ} \mathrm{C}\right)$ & 27.0 & 2.6 & 30 & 25 & 28.0 & 4.4 & 33 & 23 \\
$\mathrm{TSS}^{1}$ agua $\left({ }^{\circ} \mathrm{C}\right)$ & 30.7 & 1.2 & 32 & 30 & 30.2 & 1.8 & 32 & 28.5 \\
$\mathrm{pH}$ & 6.2 & 0.3 & 6.5 & 6 & 6.3 & 0.3 & 6.5 & 6 \\
Amonio & 0.7 & 0.1 & 0.8 & 0.6 & 0.7 & 0.1 & 0.8 & 0.6 \\
Alcalinidad $\left(\mathrm{mg} \mathrm{CaCO}_{3} / \mathrm{l}\right)$ & 68.0 & 45.1 & 120 & 40 & 52.0 & 17.4 & 72 & 40 \\
$\mathrm{CO}_{2}(\mathrm{mg} / \mathrm{l})$ & 13.7 & 4.0 & 16 & 9 & 10.5 & 1.0 & 12 & 10 \\
Cloruro & 34.0 & 19.3 & 56 & 20 & 36.0 & 0.0 & 36 & 36 \\
Dureza $(\mathrm{mg} \mathrm{CaCO} / 1)$ & 49.3 & 2.3 & 52 & 48 & 63.8 & 34.9 & 96 & 21 \\
$\mathrm{O}_{2}(\mathrm{mg} / \mathrm{l})$ & 2.8 & 2.9 & 6.1 & 0.6 & 2.4 & 1.6 & 3.8 & 0.6 \\
\hline Yarinal & & & & & & & & \\
\hline Temp. ambiental $\left({ }^{\circ} \mathrm{C}\right)$ & 27.8 & 3.4 & 32 & 25 & 27.5 & 3.0 & 30 & 24 \\
TSS ${ }^{1}$ agua $\left({ }^{\circ} \mathrm{C}\right)$ & & & & & 30.3 & 1.2 & 31 & 29 \\
$\mathrm{pH}^{\text {Amonio }}$ & 6.8 & 0.5 & 7.5 & 6.5 & 6.3 & 0.3 & 6.5 & 6.0 \\
Alcalinidad $\left(\mathrm{mg} \mathrm{CaCO}_{3} / \mathrm{l}\right)$ & 0.7 & 0.1 & 0.8 & 0.6 & 0.8 & 0.2 & 1 & 0.6 \\
$\mathrm{CO}_{2}(\mathrm{mg} / \mathrm{l})$ & 14.6 & 14.3 & 52 & 18.5 & 40.0 & 4.0 & 44 & 36 \\
$\mathrm{Cloruro}$ & 14.3 & 7.9 & 25 & 7 & 8.3 & 1.7 & 10 & 6 \\
Dureza $(\mathrm{mg} \mathrm{CaCO} / 1)$ & 31.0 & 15.1 & 52 & 20 & 30.0 & 12.2 & 38 & 16 \\
$\mathrm{O}_{2}(\mathrm{mg} / \mathrm{l})$ & 49.0 & 6.0 & 56 & 44 & 39.5 & 15.7 & 60 & 22 \\
\hline
\end{tabular}

${ }^{1}$ Temperatura sub superficial del agua

Los valores medios de $\mathrm{pH}$ caracterizan al cuerpo de agua como ligeramente ácida. Por otro lado, la dureza, en general, la tipifica como de aguas blandas, no sobrepasando los
$75 \mathrm{mg} / \mathrm{l}$ en las tres estaciones de muestreo y dos épocas. Los valores de alcalinidad sugieren que se trata de un cuerpo de agua de productividad baja, aunque variaciones loca- 
les como en Agua Blanca que llegan a presentar valores de $120 \mathrm{mg} \mathrm{CaCO}_{3} / 1$, caracterizarían a esta zona como de productividad media (Roldán, 1992; Romero, 2013).

Los resultados no muestran diferencias físico-químicas importantes con el Plan de Manejo Pesquero en la Laguna Chauya (Bazán y Deza, 2008). Asimismo, no se pueden deducir impactos antrópicos sobre las características fisicoquímicas en este cuerpo de agua con base a los registros obtenidos.

\section{Literatura Citada}

1. Bazán R, Deza S. 2008. Evaluación íctica de la laguna Chauya. En: Rios D, Riofrio J, Bazán R, Zaldívar J (eds). Plan de Manejo Pesquero en la Laguna Chauya. Ucayali, Perú: GOREU/ DIRESEPE-Ucayali/IIAP/ICBAR/ IVITA. p 86-105.

2. Esteves F. 1998. Fundamentos de limnología. $2^{\circ}$ ed. Rio de Janeiro, Brasil: Interciencia. $602 \mathrm{p}$.

3. Ramírez S, Velarde D. 2008. Estudio batimétrico de la laguna Chauya (Distrito de Masisea, Ucayali, Perú). En: Rios D, Riofrio J, Bazán R, Zaldívar J (eds). Plan de Manejo Pesquero en la Laguna Chauya. Ucayali, Perú: GOREU/ DIRESEPE-Ucayali/IIAP/ICBAR/ IVITA. $\mathrm{p}$ 49-65.

4. Riofrio J. 2002. Aspectos biométricos $y$ reproductivos de boquichico Prochilodus nigricans Agassiz, 1829 (Pisces: Prochilodontidae) en Ucayali,
Perú. Rev Peru Biol 9: 111-115. doi: 10.15381/rpb.v9i2.2529

5. Riofrio J, Clavo M, Zaldívar J, Contreras G 2008. Aportes al conocimiento y caracterización limnológica de la laguna Chauya (Distrito Masisea, Ucayali, Perú). En: Rios D, Riofrio J, Bazán R, Zaldívar J (eds). Plan de Manejo Pesquero en la Laguna Chauya. Ucayali, Perú: GOREU/DIRESEPEUcayali/IIAP/ICBAR/IVITA. p 66-77.

6. Roldán G. 1992. Fundamentos de limnología neotropical. Colombia: Universidad de Antioquía. 529 p.

7. Romero J. 2013. Calidad del agua. Colombia: Ed Escuela Colombiana de Ingeniería. $485 \mathrm{p}$.

8. Samanez I. 2008. El plancton en la laguna Chauya (Distrito de Masisea, Ucayali, Perú). En: Rios D, Riofrio J, Bazán R, Zaldívar J (eds). Plan de Manejo Pesquero en la Laguna Chauya. Ucayali, Perú: GOREU/DIRESEPEUcayali/IIAP/ICBAR/IVITA. p 78-85.

9. Sioli H. 1984. The Amazon and its main affluents: hydrography, morphology of the rivers courses, and river types. In: The Amazon. Limnology and landscape ecology of mighty tropical river and its basin. Dordrecht: Dr W Junk Publishers. p 127-165.

10. Velarde D, Ferré W, Luján G. 2008. Actividad de la flota pesquera en la laguna Chauya (Distrito Masisea, Ucayali, Perú). En: Rios D, Riofrio J, Bazán R, Zaldívar J (eds). Plan de Manejo Pesquero en la Laguna Chauya. Ucayali, Perú: GOREU/DIRESEPE-Ucayali/ IIAP/ICBAR/IVITA. p 106-121. 